\title{
Cerebrospinal Fluid Biomarkers and Normal Pressure Hydrocephalus: A Perfect Duo?
}

Keywords: normal pressure hydrocephalus, cerebrospinal fluid, biomarkers

Normal pressure hydrocephalus (NPH) is a relatively frequent but largely underdiagnosed geriatric disorder characterized by impaired gait and balance, urinary frequency, and cognitive decline with evidence of ventriculomegaly on neuroimaging. ${ }^{1}$ However, NPH pathophysiology and nosology is far from being elucidated, especially in light of the debated relationship with both neurodegenerative and vascular conditions (Figure 1). ${ }^{2,3}$ Although cerebrospinal fluid (CSF) shunting remains an effective treatment, the patient's response after diversion can be variable, sometimes of short duration, and not always predictable. Either poor patient selection or delayed surgery may explain the variability after surgery. Both of these elements represent the diagnostic difficulties in patients with features consistent with NPH.

Because an integral step in the diagnostic evaluation of NPH patients is the large-volume lumbar puncture (tap test), it makes perfect sense to examine the CSF as a potential source of diagnostic information in the hopes of improving the diagnostic yield for the diagnosis. Over the years, many biomarkers have been explored in the CSF of degenerative conditions, in some cases reaching a clinical value (Table 1$).{ }^{4}$ Several CSF studies examining for potential biomarkers have been performed in NPH patients as well. However, this literature is quite confusing because not only is NPH a heterogeneous condition, but also because these studies have explored different biomarkers, using different methodology and, more importantly, basing enrollment on a variety of different criteria. In addition, CSF biomarker levels potentially can be altered through possible changes of intracranial fluid dynamics for the clearance of proteins in patients with NPH. ${ }^{5}$

In this issue of The Canadian Journal of Neurological Sciences, Pfanner et al present the result of their systematic review of studies investigating CSF biomarkers as predictors of shunt response in patients with idiopathic $\mathrm{NPH}^{6}$ The authors should be congratulated not only for an extremely timely review, but also for their conservative approach to avoid misdiagnoses that could bias CSF results. They decided to focus on selected studies with an adequate sample ( $>$ ten cases) in which "idiopathic" and "secondary" NPH were not combined, and on patients with a documented improvement after CSF shunting. Authors were left with 13 studies with data on 508 patients and 37 unique CSF biomarkers. They concluded that the available evidence is greatest in predicting shunt response in idiopathic NPH for amyloid beta peptide 42, tau, phosphorylated tau, neurofilament light protein (NFL), and leucine-rich alpha-2-glycoprotein (LRG).

Decreased CSF levels of amyloid beta peptide 42, and increased CSF levels of tau and phosphorylated tau, are strongly

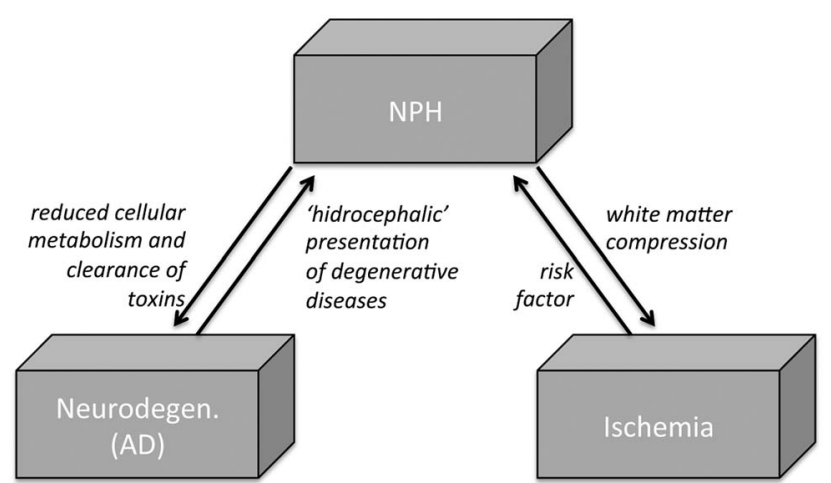

Figure 1: The debated relationship among NPH, neurodegeneration, and vascular conditions. In NPH, CSF flow is pushed through the Virchow-Robin spaces into brain parenchyma, resulting in parenchymal changes from tissue compression and white matter ischemia. On the other hand, some vascular risk factors (hypertension and diabetes) have been linked to an increased risk of NPH. In addition, venous compliance in NPH is found to be reduced, particularly in the sagittal sinus, possibly resulting in reduced cellular metabolism and clearance of toxins, thus favoring AD-like neurodegenerating. $A D=$ Alzheimer disease; $C S F=$ cerebrospinal fluid; $\mathrm{NPH}=$ normal pressure hydrocephalus .

associated with Alzheimer disease (AD) ${ }^{4,7}$ Not surprisingly, NPH patients with biomarker profiles consistent with $\mathrm{AD}$ tended toward lower postoperative improvement. ${ }^{6}$ More complex is the interpretation of NFL and LRG, markers of neuronal damage and inflammation, respectively. Both markers are significantly elevated in NPH patients, but their predictive role is complicated by the fact that patients with longstanding NPH might not respond to shunting when surgery is performed too late, in spite of elevated NFL and LRG.

The review by Pfanner et al. ${ }^{6}$ also found "minimal evidence" for the use of transforming growth factor- $\beta 1$, transforming growth factor beta receptor 2 , homocysteine, and interleukins $1 \beta, 6$, and 10. The authors also identified other CSF biomarkers that are commonly investigated in neurological conditions that have not been investigated for NPH. These include $\alpha$-synuclein, interferon gamma, neuron-specific enolase, and visinin-like protein-1.

The overall interpretation of the scientific evidence collected so far further indicates that NPH is a syndrome rather than a discrete entity with a diagnostic gold standard. In this context, although limited by the lack of detailed clinical information,

Received August 13, 2017. Date of Acceptance August 17, 2017. 
Table 1: Principal CSF biomarkers profile in principal dementia syndromes (modified from ${ }^{6}$ ).

\begin{tabular}{|c|c|c|c|c|c|}
\hline & $\mathbf{A \beta} 42$ & Tau & p-Tau & LNF & Others \\
\hline $\mathrm{AD}$ & $\downarrow$ & $\uparrow$ & $\uparrow$ & $\uparrow *$ & $\begin{array}{l}\alpha \text {-synuclein: } \uparrow / \mathrm{N}^{*} \\
\text { CHI3L1: } \uparrow^{*} \\
\text { D-Serine: } \uparrow^{*} \\
\text { Ferritin: } \uparrow^{*} \\
\text { mDNA: } \downarrow^{*} \\
\text { miR-27-a-3p; miR-34a; miR-125b; mIR-146a: } \downarrow^{*} \\
\text { mIR-29: } \uparrow^{*} \\
\text { Neurogranin: } \uparrow^{*} \\
\text { sAPP: } \uparrow^{*} \\
\text { SNAP-25: } \uparrow^{*} \\
\text { Metabolomics }\end{array}$ \\
\hline $\mathrm{FTD}^{\ddagger}$ & $\downarrow / \mathrm{N}^{*}$ & $\uparrow$ & $\uparrow / N^{*}$ & $\uparrow$ & $\begin{array}{l}\text { NFH: } \uparrow * \\
\text { TDP-43: } \uparrow / N^{*}\end{array}$ \\
\hline $\mathrm{PDD} / \mathrm{LBD}$ & $\downarrow$ & $\downarrow^{*}$ & $\mathrm{~N}^{*}$ & $\uparrow$ & $\alpha$-synuclein: $\downarrow$ \\
\hline VD & $\downarrow / \mathrm{N}$ & $\uparrow / \mathrm{N}$ & $\uparrow / \mathrm{N}$ & $\uparrow^{*}$ & A $\beta 40: \downarrow$ \\
\hline CJD & $\downarrow$ & $\uparrow$ & $\uparrow$ & $\uparrow^{*}$ & $\begin{array}{l}14-3-3 \uparrow \\
\alpha \text {-synuclein: } \downarrow \\
\operatorname{PrP}, \operatorname{PrPc}: \downarrow \\
\text { PrPsc: } \uparrow\end{array}$ \\
\hline
\end{tabular}

Abbreviation: $\downarrow=$ reduced; $\uparrow=$ increased; $\mathrm{A} \beta=$ amyloid beta; $\mathrm{AD}=$ Alzheimer disease; CHI3L1 = Chitinase-3-like protein (or YKL-40); CJD =CreutzfeldJacob Disease; $\mathrm{CSF}=$ cerebrospinal fluid $; \mathrm{FTD}=$ fronto-temporal dementia; $\mathrm{LBD}=$ Lewy body dementia; $\mathrm{mDNA}=$ mitochondrial $\mathrm{DNA} ;$ miR $=$ microRNA; $\mathrm{N}=$ normal; $\mathrm{NFH}=$ neurofilament heavy protein; $\mathrm{NFL}=$ neurofilament light protein; $\mathrm{PDD}=$ Parkinson's disease-dementia; $\operatorname{Prp}=\operatorname{Prion}$ protein; PrPc = cellular prion protein; PrPsc = conversion of PrPc to scrapie prion protein; $\mathrm{p}$-tau = phosphorylated tau at threonine-181; $\mathrm{sAPP}=$ soluble amyloid precursor protein; $\mathrm{VD}=$ vascular dementia.

*Further studies required.

${ }^{\dagger}$ Tyrosine, purine, tocopherol, choline, valine, arginine, suberylglycerine, carnitine, creatine, histidine, cholesterol, and sphingolipids are under investigation. ${ }^{\ddagger}$ No reliable CSF biomarker because of pathological heterogeneity.

variable follow-up duration, and overall poor quality of the studies under scrutiny, this systematic review by Pfanner et $\mathrm{al}^{4}$ identifies a possible clinical value for biomarkers in NPH, particularly in light of their role in diagnosing AD.

\section{ACKNowledgments AND Funding}

AF reports grants from University of Toronto, the McLaughlin Centre, and the Michael J. Fox Foundation; personal fees from UCB pharma, Medtronic, Boston Scientific, Abbvie, Novartis, Chiesi pharmaceutical; and TEVA, grants from Medtronic, and Boston Scientific, Abbvie outside the submitted work.

\section{Disclosures}

The authors have nothing to disclose.

\section{Statement of Authorship}

$\mathrm{AF}$ and DFTW conceived the article and undertook manuscript drafting.

Alfonso Fasano

Department of Medicine (Neurology), University of Toronto

Toronto, Ontario, Canada

Morton and Gloria Shulman Movement Disorders Centre and the Edmond J. Safra Program in Parkinson's Disease University Health Network, Toronto Western Hospital Toronto, Ontario, Canada Krembil Research Institute, Toronto, Ontario, Canada
David F. Tang-Wai Department of Medicine (Neurology), University of Toronto Toronto, Ontario, Canada Krembil Research Institute, Toronto, Ontario, Canada University Health Network Memory Clinic, Toronto Western Hospital, Toronto, Ontario, Canada

Correspondence to: Alfonso Fasano, Department of Medicine Suite RFE 3-805, 200 Elizabeth Street

Toronto, ON, M5G $2 \mathrm{C} 4$ Email: alfonso.fasano@uhn.ca

\section{REFERENCES}

1. Fasano A, Bloem BR. Gait disorders. Continuum. 2013;19: 1344-1382.

2. Cabral D, Beach TG, Vedders L, et al. Frequency of Alzheimer's disease pathology at autopsy in patients with clinical normal pressure hydrocephalus. Alzheimers Dement. 2011;7:509-13.

3. Starr BW, Hagen MC, Espay AJ. Hydrocephalic parkinsonism: lessons from normal pressure hydrocephalus mimics. J Clin Mov Disord. 2014;1:2.

4. Llorens F, Schmitz M, Ferrer I, Zerr I. CSF biomarkers in neurodegenerative and vascular dementias. Prog Neurobiol. 2016; 138-140:36-53.

5. Graff-Radford NR. Alzheimer CSF biomarkers may be misleading in normal pressure hydrocephalus. Neurology. 2014;83:1573-5.

6. Pfanner T, Henri-Bhargava A, Borchert S. CSF biomarkers as predictors of shunt response in idiopathic normal pressure hydrocephalus. Can J Neurol Sci. In press.

7. Olsson B, Olsson C, Lautner R, et al. CSF and blood biomarkers for the diagnosis of Alzheimer's disease: a systematic review and meta-analysis. Lancet Neurol. 2016;15:673-84. 